\title{
Pre-brachytherapy magnetic resonance- based 3D tumor volume evaluation for outcome prediction in cervical cancer patients treated with definitive chemoradiation
}

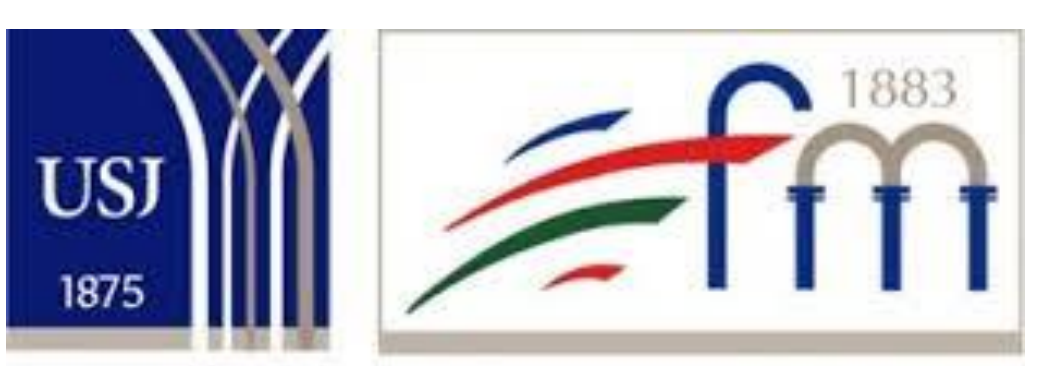

Université Saint-Joseph de Beyrouth Faculté de médecine

Clement El Khoury ${ }^{1}$, David Atallah², Joy $\mathrm{Naba}^{3}$, Aref Nassar ${ }^{3}$, Malak Moubarak², Fares Azoury ${ }^{1}$, Tony Felefly ${ }^{1}$, Jad El Barouky ${ }^{1}$, Rima Sayah ${ }^{1}$, Dolly Nasr ${ }^{1}$, Georges Chahine ${ }^{4}$ and Elie Nasr ${ }^{1}$.

1 University of Saint Joseph, Hotel Dieu de France - Department of Radiation Oncology, Beirut, Lebanon.

2University of Saint Joseph, Hotel Dieu de France - Department of Gynecology - Division of Gynecologic Oncology, Beirut, Lebanon.

${ }^{3}$ University of Saint Joseph, School of Medicine, Beirut, Lebanon.

${ }^{4}$ University of Saint Joseph, Hotel Dieu de France - Department of Medical Oncology, Beirut, Lebanon.

Objectives: To report the impact on survival of residual tumor volume (RTV) prior to brachytherapy (BT) initiation determined by magnetic resonance imaging (MRI) in patients treated for locally advanced cervical cancer with definitive chemoradiotherapy (CRT).

Methods: MRI examinations were prospectively performed in patients with advanced cervical cancer (Stages IB2-IVA) after CRT completion and prior to BT initiation. RTV was delineated on each T2-weighted slice and volumetry was derived on the treatment planning system. All patients received external beam radiation (EBRT) with concomitant Cisplatin followed by volumebased BT planning. Cumulative EBRT and BT doses were calculated as the total equivalent dose in 2 Gy fractions (EQD2). Potential prognostic factors were selected based on non-parametric tests and then analyzed for survival with a Cox regression model.

Results: Median post-therapy follow-up was 27.4 months (range, 3-57.8). Thirty-seven patients were included. According to the FIGO classification, $8 \%$ were stage IB, $75 \%$ stage II, $11 \%$ stage III, and $6 \%$ stage IV. Overall survival was $86.5 \%$. Of the patients evaluated, $29.7 \%$ had complete remission on pre-BT MRI and $18.9 \%$ were considered having local failure or distant disease. At bivariate analysis, RTV >2 mL, D90 CTV-HR $<84$ Gy and excessive treatment time were all predictors of poor overall survival.
At multivariate regression analysis, only RTV persisted as a significant prognostic factor with survival rates of $95.8 \%$ and $69.2 \%$ for pre-BT RTV $\leq 2 \mathrm{~mL}$ and $>2 \mathrm{~mL}$ respectively $(p=0.005)$.

Conclusion: Our preliminary data suggest that pre-BT $\mathrm{RTV}>2 \mathrm{~mL}$ is a predictor of poor survival.

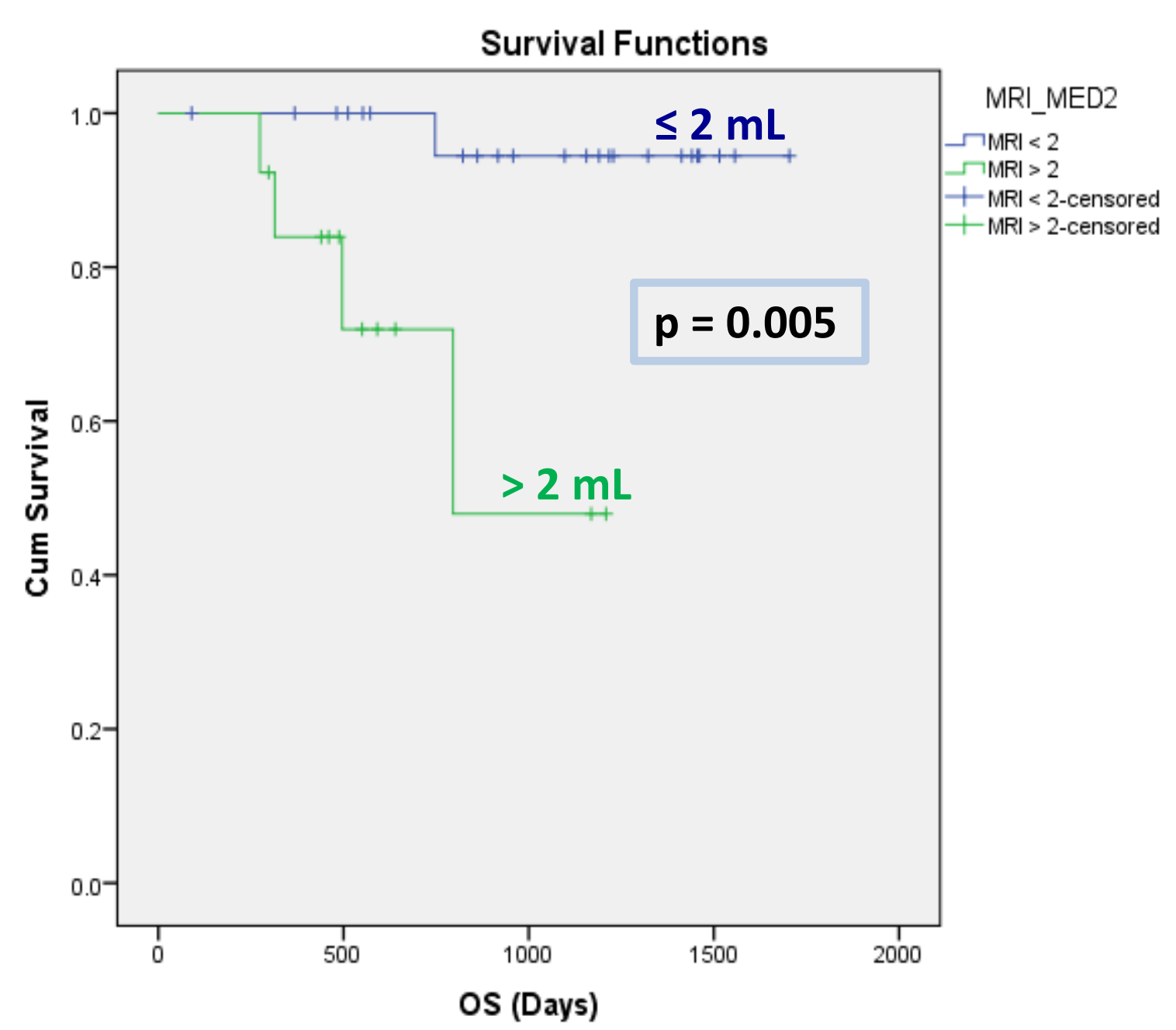

Estimates of overall survival in patients with cervical cancer after definitive CRT with $R T V \leq 2 \mathrm{~mL}$ or $>2 \mathrm{~mL}$.

\begin{tabular}{|r|r|r|}
\hline Pre-BT RTV & $\leq 2 \mathrm{~mL}$ & $>2 \mathrm{~mL}$ \\
\hline OS & $95.8 \%$ & $69.2 \%$ \\
\hline $\mathrm{p}$ & 0.005 & \\
\hline
\end{tabular}

Comparison of survival using pre-BT RTV 